\title{
Test-beam performance of a TORCH prototype module
}

\author{
M. Kreps $^{a, *}$, S. Bhasin ${ }^{b, c}$, T. Blake ${ }^{a}$, N.H. Brook ${ }^{b}$, M.F. Cicala ${ }^{a}$, T. Conneely ${ }^{d}$, \\ D. Cussans ${ }^{c}$, M.W.U. van Dijk ${ }^{e, 1}$, R. Forty ${ }^{e}$, C. Frei $^{e}$, E.P.M. Gabriel $^{f}$, R. Gao ${ }^{g}$,

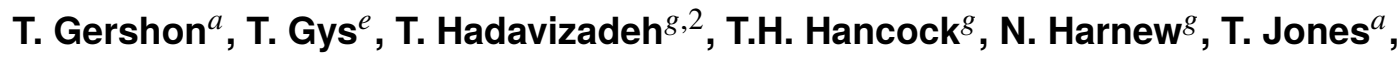 \\ J. Milnes $^{d}$, D. Piedigrossi ${ }^{e}$, J. Rademacker ${ }^{c}$, J. Smallwood $^{g}$
}

${ }^{a}$ Department of Physics, University of Warwick, Coventry, UK

${ }^{b}$ University of Bath, Claverton Down, Bath, UK

${ }^{c}$ H.H. Wills Physics Laboratory, University of Bristol, Tyndall Avenue, Bristol, UK

${ }^{d}$ Photek Ltd., 26 Castleham Road, St Leonards on Sea, UK

${ }^{e}$ CERN, Geneva, Switzerland

${ }^{f}$ School of Physics and Astronomy, University of Edinburgh, James Clerk Maxwell Bldg., Edinburgh, UK

${ }^{g}$ Denys Wilkinson Laboratory, University of Oxford, Keble Road, Oxford, UK

E-mail: michal.kreps@cern.ch

The TORCH time-of-flight detector is designed to provide a $15 \mathrm{ps}$ timing resolution for charged particles, resulting in $K / \pi(p / K)$ particle identification up to momentum of about $10(15) \mathrm{GeV} / c$ over a $10 \mathrm{~m}$ flight distance. Cherenkov photons, produced in a quartz plate of $10 \mathrm{~mm}$ thickness, are focused onto an array of micro-channel plate photomultipliers (MCP-PMTs) which measure the photon arrival times and spatial positions. A TORCH demonstrator module instrumented with a customised MCP-PMTs has been tested at the CERN PS. The useful implementation for the particle identification in the $\mathrm{LHCb}$ experiment requires single-photon time resolution of $70 \mathrm{ps}$. The timing performance and photon yields have been measured as a function of beam position in the radiator, giving measurements which are approaching the required resolution. A possible TORCH design of the particle identification system in the LHCb experiment has been simulated and its potential for high luminosity running has been evaluated.

40th International Conference on High Energy physics - ICHEP2020

July 28 - August 6, 2020

Prague, Czech Republic (virtual meeting)

\footnotetext{
${ }^{1}$ Now at École Polytechnique Fédérale de Lausanne (EPFL), Laboratoire de Physique des Hautes Energies, Cubotron UNIL, Route de la Sorge, CH-1015 Lausanne, Switzerland

${ }^{2}$ Now at School of Physics and Astronomy, Monash University, Melbourne, Australia

*Speaker
} 


\section{Introduction}

The success of the physics programme of the LHCb experiment heavily relies on excellent particle identification (PID) capabilities. From the start of the data taking, PID was performed using Ring Imaging Cherenkov (RICH) detectors [1]. With the existing RICH detectors, momentum threshold for the kaons below which no light is emitted is about $10 \mathrm{GeV} / c$. For the protons, the threshold is even higher and thus LHCb detector does not provide positive proton identification for tracks with momentum below $15 \mathrm{GeV} / c$. Adding PID capabilities in the lower momentum region, has potential to significantly improve sensitivity across a wide physics programme of the experiment.

A possible option to extend PID capabilities of the LHCb experiment is based on the timeof-flight measurement over a distance of about $10 \mathrm{~m}$. The difference in the time-of-flight between pions and kaons with the momentum of $10 \mathrm{GeV} / \mathrm{c}$ is about $35 \mathrm{ps}$, which translates to the requirement for per track time resolution of about $10-15 \mathrm{ps}$. The work presented in these proceedings aims to demonstrate that this is achievable using detector based on the timing of Cherenkov photons emitted in the $1 \mathrm{~cm}$ thick quartz bar. The TORCH detector for $\mathrm{LHCb}$ experiment based on this principle would consist of 18 modules, each being composed of $250 \times 66 \times 1 \mathrm{~cm}^{3}$ quartz bar equipped with focusing block with cylindrical mirror and 11 microchannel plate (MCP) PMTs as photodetectors $[2,3]$. The basic principle is that the Cherenkov photons propagate through the quartz bar via total internal reflection into focusing block, where they are focused in one direction. With such setup, about 20-30 photons per track are expected to be detected, which for desired per track time resolution translates to the time resolution of about $70 \mathrm{ps}$ for the single photons. Of that, about $50 \mathrm{ps}$ would be intrinsic time resolution of the photodetector and about $50 \mathrm{ps}$ would come from the electronics used.

The MCP-PMTs are developed in an R\&D programme with a commercial partner Photek Ltd. [4]. The main aim of the development was to provide PMTs with a long lifetime and high granularity. The developed device has an active area of $53 \times 53 \mathrm{~mm}^{2}$ in the housing with a size of $60 \times 60 \mathrm{~mm}^{2}$. Internally, PMT has $64 \times 64$ pixels with the baseline for TORCH being that in non-focusing direction, 8 pixels are grouped together, but the design would allow grouping fewer pixels if higher granularity is needed. The effective resolution in the focusing direction is improved by charge sharing where photon would be typically detected on more than one neighbouring pixels.

The goal of the test beam studies is to demonstrate that the timing of the Cherenkov photons can be achieved with required single-photon resolution of $70 \mathrm{ps}$. In this work, we will show that the project made significant progress towards this goal and that the required resolution is within reach.

\section{Test beam setup}

The test beam campaign took place in autumn 2018 at CERN at proton synchrotron (PS) using East area T9 beamline, which provided mixed pion-proton beam with a momentum of $5 \mathrm{GeV} / c$. The TORCH module used had quartz bar of size $125 \times 66 \times 1 \mathrm{~cm}^{3}$, which is half-size of the module needed for the LHCb. The module was instrumented with 2 MCP PMTs which were mounted next to each other on the edge of the module. The readout [5] is based on NINO [6] and HPTDC [7] chips, which provide a time when the signal passes threshold and time over the threshold. To define beam and its 

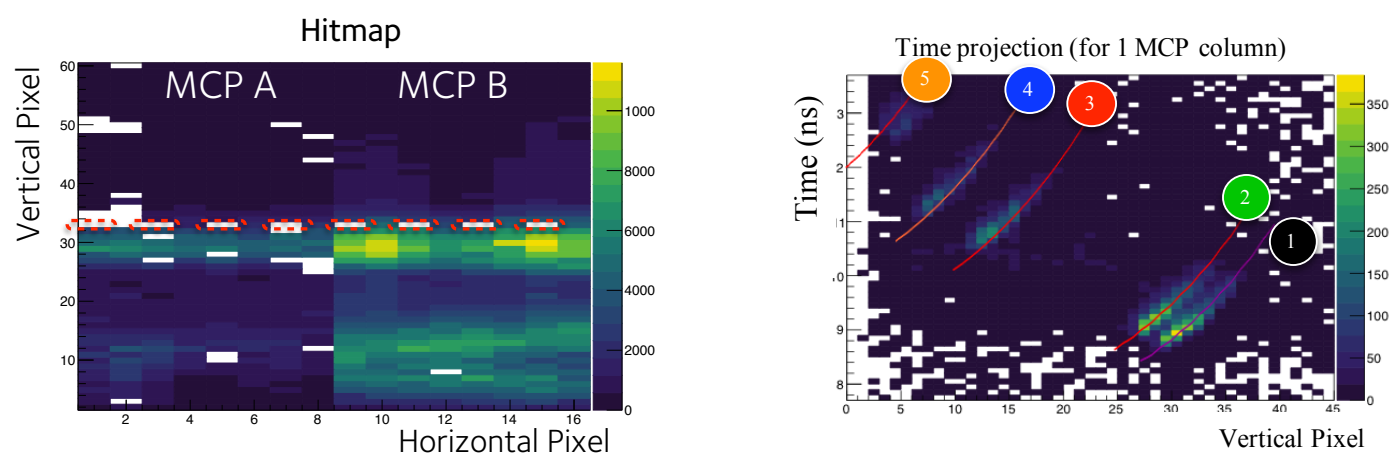

Figure 1: The distribution of the photon hits in the spatial coordinates on the MCP-PMTs (left) and the distribution of the photon hits along vertical pixel (finer segmented direction) and photon arrival time for the single MCP-PMT column (right). The marked bands show the expectation for the photon positions and arrival time with each band corresponding to the different path in the quartz radiator with band 1 having the smallest number of reflections along the long side of the radiator.

timing, several additional detectors are used. At the entrance to the area, two Cherenkov counters are used to identify hadrons in the beam. In front of the tested TORCH prototype, a EUDET/AIDA pixel beam telescope [8] is installed to measure the beam profile incident on TORCH. The beam definition is complemented by two timing stations spaced approximately $11 \mathrm{~m}$ apart to provide time reference. In Fig. 1 the TORCH image is shown. In spatial coordinates, the image follows the typical Cherenkov light ring, which is folded by the reflections on side of the quartz bar. Looking at a single column, as one moves over pixels the signal is detected at different times. The various bands observed can be attributed to the fixed number of reflections along the long side of the quartz bar. It should be noted that the two MCP-PMTs used in the test beam had different quantum efficiency, which is responsible for the difference in the number of detected photons seen in Fig. 1.

\section{Results}

The requirement on per-track resolution relies on achieving single-photon resolution of $70 \mathrm{ps}$ and detection of a sufficient number of photons per track. Therefore, it is important to test how well the number of observed photons is predicted. The check is done with different beam entry points into the radiator going from $17.5 \mathrm{~cm}$ from the top of the quartz bar to $111 \mathrm{~cm}$. In Fig. 2 the distribution of the number of hits detected in both MCP-PMTs in data is compared to the simulation, while in table 1 the mean number of hits is compared. One should recall that the prototype is instrumented with just 2 MCP-PMTs out of 11 and that one of them had a quantum efficiency well below the expectation, so the numbers are not a direct representation of the complete module. Overall, a good agreement is observed with a small discrepancy at the beam position closest to the MCP-PMTs which is mainly due to events without any hit observed.

To extract the MCP-PMT time resolution, the photons with the lowest number of reflections along the long side of the radiator are selected. For these, the time distributions on the individual pixels in a single column of 64 pixels are fitted simultaneously. The shape is described by a Gaussian core which transitions into a power-law tail. The power-law tail captures the signal due to the electrons backscattered on the MPC-PMTs anode. An example from the simultaneous fit for the 

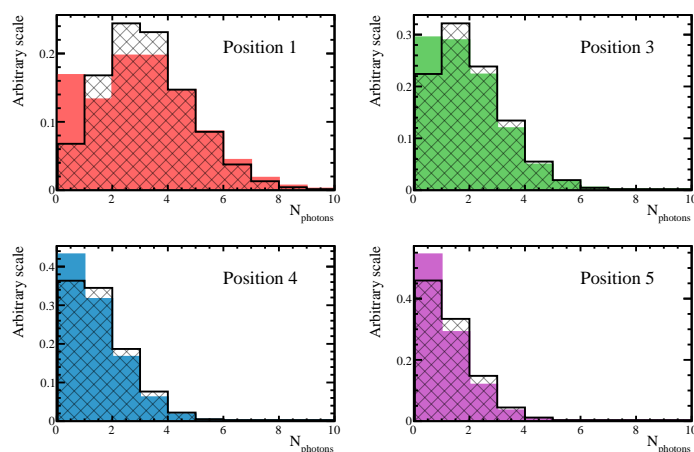

Figure 2: Distribution of number of observed hits per track in data (shaded) and simulation (solid) for different beam entry points.

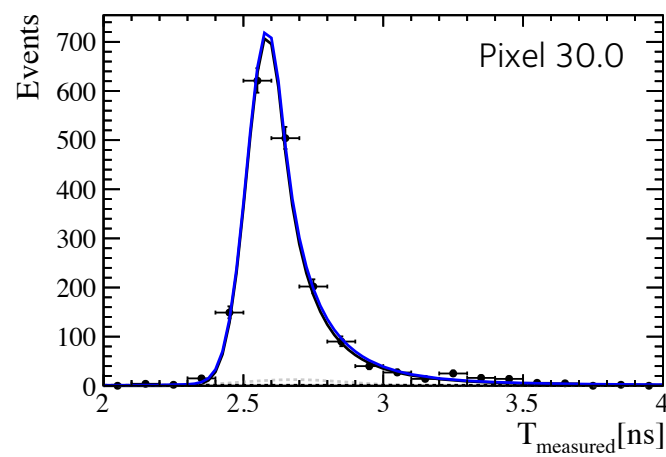

Figure 3: Distribution of the photon arrival times on a single pixel of MCP-PMT (points) with fit to extract time resolution overlayed (blue line).

Table 2: The fit parameters for the parametrisation of

Table 1: Comparison of the mean number of observed hits between data and simulation for various beam entry positions.

\begin{tabular}{cccc} 
Position & Data & Simulation & Ratio \\
\hline 1 & 2.60 & 2.73 & 1.05 \\
3 & 1.42 & 1.56 & 1.10 \\
4 & 0.94 & 1.07 & 1.14 \\
5 & 0.68 & 0.82 & 1.22
\end{tabular}
the time resolution on the photon propagation time and cluster size along with the expectation corresponding to the required performance.

\begin{tabular}{ccc} 
& Expectation [ps] & Measurement [ps] \\
\hline$\sigma_{\text {const }}$ & 33 & $33.0 \pm 7.1$ \\
$\sigma_{\text {prop }}^{0}$ & $3.75 \pm 0.8$ & $8.8 \pm 0.7$ \\
$\sigma_{\text {RO }}^{0}$ & 60 & $100.5 \pm 5.7$
\end{tabular}

individual pixel is shown in Fig. 3. The extracted resolution for the different columns and various beam incident positions is also shown in Fig. 4. The dependence on the position in the MCP-PMT is small and variations can be attributed to the imperfect electronics calibration and selection of the photons for the analysis.

The dependence on the entry point to the quartz bar translates to the dependence on the pathlength of the photon inside the bar. As can be seen from the Fig. 5, the resolution can be parametrised as a function of photon propagation time and number of pixels within the cluster as

$$
\sigma_{\mathrm{TORCH}}^{2}=\sigma_{\text {const }}^{2}+\sigma_{\text {prop }}^{2}(t)+\sigma_{\mathrm{RO}}^{2}\left(N_{\text {pixels }}\right) .
$$

Assuming that the $\sigma_{\text {prop }}(t)=\sigma_{\text {prop }}^{0} t[\mathrm{~ns}]$ and that $\sigma_{\mathrm{RO}}=\sigma_{\mathrm{RO}}^{0} / \sqrt{N_{\text {pixels }}}$, the resolutions in Fig. 4 can be parametrized by single set of parameters, which are listed in table 2 . While the measured values are somewhat worse than the expectation, the analysis is not yet completed and has room for improvement. The most important aspect is the calibration needed. The exact timing of the signal depends on the signal strength and electronics reading out individual pixels. Corresponding effects are currently calibrated on the test beam data exploiting time-over-threshold information. While reasonable calibration can be achieved, dedicated calibration of the electronics with well-defined signals will yield improved calibration. The effect has been tested in a laboratory environment by illuminating a single pixel of the MCP-PMT with a fast pulsed laser and reading out signals. The analysis can then concentrate on the signals of the same width to remove spread due to time-walk 


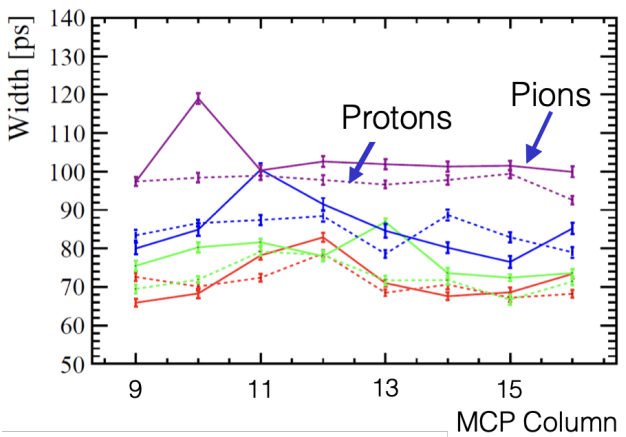

Figure 4: The measured time resolution for individual columns on the MCP-PMT. The different colours represent different beam entry points with red being closest to the MCP-PMT, while the full line shows measurement with pion beam while the dashed one uses proton beam.

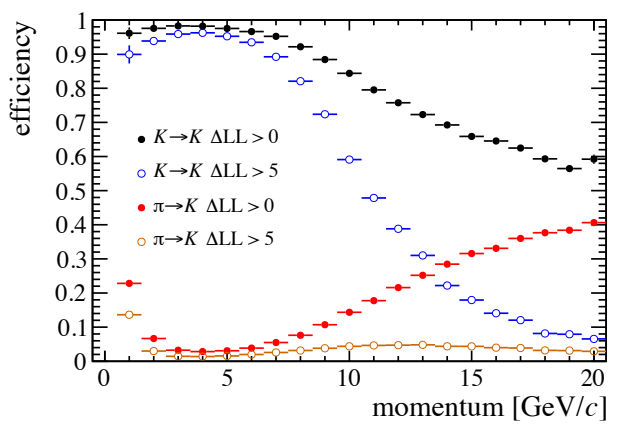

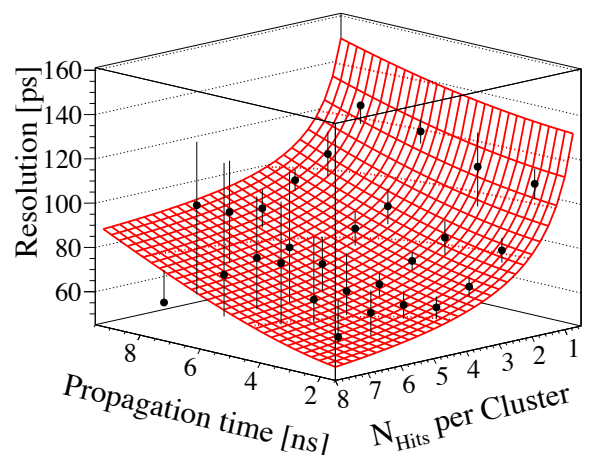

Figure 5: The dependence of the measured time resolution on the photon propagation time and the cluster size. The mesh represents a fit with linear function in propagation time and the inverse of the square root of the cluster size.

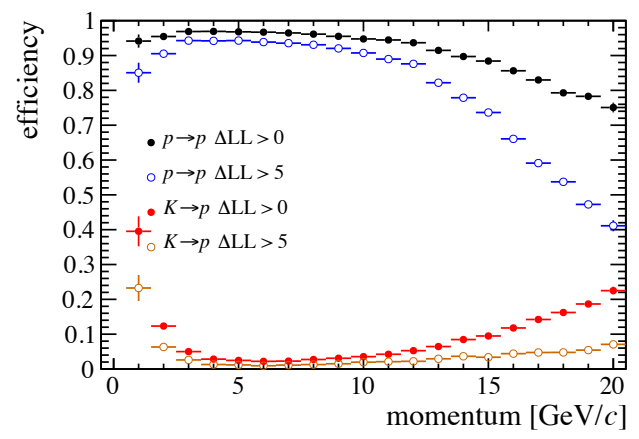

Figure 6: Particle identification efficiency for kaons (left) and protons (right) with two different requirements on the negative log-likelihood difference of observing the image given the particle hypothesis. The misidentification rate of pions as kaons (left) and kaons as protons (right) is also shown.

effects. In the setup, the HPTDC is operated in $25 \mathrm{ps}$ mode compared to the $100 \mathrm{ps}$ mode in the test beam and the resolution of $49.6 \mathrm{ps}$ was measured which compares well with the expectation of $50 \mathrm{ps}$. This indicates that with improvements in calibrations, significant improvement is expected and suggests that the design performance is achievable.

As part of the motivation is to improve low momentum particle identification at $\mathrm{LHCb}$, it is useful to evaluate such performance. For this, TORCH detector is added to the Geant 4 based simulation [9-11] of LHCb in Run 3 of the LHC accelerator. The ability to separate kaons from pions and protons from kaons at lower momentum is demonstrated in Fig. 6, where the selection based on the likelihood ratio between different particle identification hypotheses is used. As can be seen, good separation for kaons from pions is achieved below momentum of $10 \mathrm{GeV} / c$ and between kaons and protons up to about $15 \mathrm{GeV} / c$ momentum. These momentum regions complement the particle identification provided by the RICH detectors and thus TORCH has great potential to improve the physics reach of the $\mathrm{LHCb}$ experiment. 


\section{Summary}

Results from the TORCH prototype test beam campaign in 2018 were presented. These show encouraging results and suggest that with additional work on the calibrations the required singlephoton resolution of $70 \mathrm{ps}$ is achievable. The dedicated measurements in the lab provide additional support that the required performance is within reach. Evaluation of the possible TORCH based time-of-flight detector considered for the LHCb detector upgrade shows that significant enhancement of the particle identification capabilities is possible. The work continues with plans for further test beam campaign with fully instrumented prototype when the CERN accelerators restart after the long shutdown, to provide the final demonstration of the concept.

\section{References}

[1] LHCв RICH GRoup collaboration, Performance of the LHCb RICH detector at the LHC, Eur. Phys. J. C 73 (2013) 2431 [1211. 6759].

[2] LHCв collaboration, TORCH: Time of Flight Identification with Cherenkov Radiation, Nucl. Instrum. Meth. A 639 (2011) 173 [1009.3793].

[3] N. Brook et al., Testbeam studies of a TORCH prototype detector, Nucl. Instrum. Meth. A 908 (2018) 256 [1805 . 04849].

[4] T. Conneely et al., The TORCH PMT: a close packing, multi-anode, long life MCP-PMT for Cherenkov applications, JINST 10 (2015) C05003.

[5] R. Gao et al., Development of TORCH readout electronics for customised MCPs, JINST 11 (2016) C04012.

[6] F. Anghinolfi, P. Jarron, A. Martemyanov, E. Usenko, H. Wenninger, M. Williams et al., NINO: An ultra-fast and low-power front-end amplifier/discriminator ASIC designed for the multigap resistive plate chamber, Nucl. Instrum. Meth. A 533 (2004) 183.

[7] M. Mota, J. Christiansen, S. Debieux, V. Ryjov, P. Moreira and A. Marchioro, A flexible multi-channel high-resolution time-to-digital converter asic, in 2000 IEEE Nuclear Science Symposium. Conference Record (Cat. No.00CH37149), vol. 2, pp. 9/155-9/159 vol.2, 2000.

[8] I. Rubinskiy and H. Perrey, An EUDET/AIDA Pixel Beam Telescope for Detector Development, PoS TIPP2014 (2014) 122.

[9] Geant4 collaboration collaboration, Geant4 developments and applications, IEEE Trans.Nucl.Sci. 53 (2006) 270.

[10] Geant4 Collaboration collaboration, Geant4: A simulation toolkit, Nucl. Instrum. Meth. A506 (2003) 250.

[11] M. Clemencic et al., The LHCb simulation application, Gauss: Design, evolution and experience, J. Phys. Conf. Ser. 331 (2011) 032023. 